\title{
'They just said come in for a day': patients' experiences of day case surgery
}

Gail P Mooney Carmarthenshire NHS Trust and School of Health Science, University of Wales, Swansea and Anthea Symonds Department of Social Policy and Applied Social Studies, University of Wales, Swansea, UK

\begin{abstract}
This paper is based on a qualitative study that was designed to investigate patients' experiences of day case surgery and its outcomes. The study was a part requirement of a Masters course in Community Care Studies, and was conducted in a Welsh Integrated Trust in 1997. A case-study approach employing semi-structured interviews was used. A total of 16 patients were interviewed (5 females and 11 males). Three of the males and all five females had varicose vein repair, and the other eight males had a hernia repair. The findings of the study were that although patients preferred the option of day care surgery and indicated that they would choose this in future, the information given to them and the postoperative care they received were largely inadequate. The interviews demonstrate that the communication between hospital and home care was often inadequate, and that some patients and carers experienced unforeseen difficulties and distress.
\end{abstract}

Key words: day case surgery; patients' experiences of day case surgery; patient information

\section{Introduction}

The phenomenon of day case surgery within the NHS is a practice introduced to 'improve patient care'. It is often said to be what patients want: 'into hospital in the morning and home in the evening', to "be cared for by their families in the community'. This paper describes a closer examination of this assumption of patient satisfaction.

Although various reports (Royal College of Surgeons of England, 1985; Department of Health, 1989; NHS Management Executive, 1993) extol the benefits of day case surgery, it would appear that the actual attitudes, fears and expectations of patients have rarely been elicited.

\section{Benefits of day case surgery - to whom?}

Since the Royal College of Surgeons of England (RCS) stated that: 'up to $50 \%$ of all post-operative

Address for correspondence: Gail P Mooney, Prince Philip Hospital, Dafen, Llanelli, Carmarthenshire SA14 8QF, UK.

Email: g.mooney@swansea.ac.uk

(C)Arnold 2001 patients need not stay in hospital overnight' (Royal College of Surgeons of England, 1985: 3-4), trends towards day care surgery have increased. The increase in the number of day cases, in which patients are admitted, treated and discharged from hospital all in one day, continues. In the first half of 1995-96, there were 1.3 million day cases, compared with 1.1 million over the same period in 1994-95, an increase of 17\% (NHS, 1995). In Wales in 1993-94 there were 201410 surgery day cases recorded, which increased to 309737 in 1997-98, representing a rise of 54\% in 4 years (Welsh Office, 1999).

The Bevan Report (Department of Health, 1989) supported the concept of day surgery and concluded that the costs of treating patients as day cases were demonstrably lower than those of treating them as in-patients. Fawcett-Henessy (1995) reported that in 1990, £31 million was saved on hernia repairs alone. Other authors have also documented such savings: 'Day case surgery can offer an appropriate and cost-effective method of treatment for a number of surgical procedures' (Bates and Hamm, 1989). It has also been suggested that 1463-4236(2001)PC032OA 
day case surgery can have other benefits for patients in that they are treated sooner, are absent from home for a much shorter period, and there is an improvement in the level of care due to the specialist facilities (James, 1993).

Avis (1992) conducted a study designed to explore patients' attitudes to choice in the context of hernia repair in a day care unit. In all cases the main determinant for the decision was a deference to professional opinion, rather than active decision making in a consumerist mode. The author states that 'this has serious implications for the formulation of a consumer model - which would give patients the opportunity to make an active and informed decision on surgery' (Avis, 1992: 61). It appeared that patients expected direction from the medical profession, because 'they know best - they are the experts'.

\section{Selection of patients for day case surgery}

Patients are usually selected for day case surgery during the out-patient consultation. Selection is dependent on a number of criteria, the primary one being the nature of the operation itself. An assessment is also made of their general physical condition in relation to general anaesthesia (Royal College of Surgeons of England, 1985). In addition, of course, the social circumstances of the patient should be investigated in order to ascertain whether they will be able to cope with their recovery once they go home. It is expected that all patients who require day case surgery will have a responsible adult to collect them from the unit and remain with them overnight. This expectation is based on the assumption that the patient has someone at home to care for them, or that they know someone who is willing to take on this role. Carrington (1993) suggests that discharge is an important part of the event, involving both patients and relatives as they take responsibility for the patient's care at home. They must be given specific advice and instructions on what to do, what to look for, and who to contact if the patient becomes unwell or worried.

\section{Pre-operative information}

Both the transmission of information from professionals to patients and its interpretation are very problematic areas to analyse. Professionals often feel that they have given full information, but patients - because of heightened levels of tension and anxiety - frequently do not 'receive' this information fully. 'Patients' "need to know and understand" may lead to additional information-seeking about what has just been told' (Ong et al., 1995). Preoperative visits are now common procedures in many hospitals (Copp, 1988; Webb, 1995). Preadmission clinics are also being set up, at which nursing staff and doctors are able to inform the patient prior to their admission for day surgery about the procedures involved and the possible outcomes of treatment, so that the patient knows what to expect. Studies have shown a clear link between the level of preoperative information given to patients and the nature of their recovery (Bysshe, 1988).

\section{Aim of the study}

The aim of the study described in this paper was to elicit patients' personal views about day case surgery, i.e., to find out whether it was what they wanted, whether they would choose day surgery again, and what was good and/or bad about their experiences.

\section{Methods}

\section{Sample population}

The population sample consisted of patients who had undergone day case surgery, in that they came into the day unit, had their operation requiring general anaesthetic and then went home on the same day. The operations that are most frequently performed in day surgery are hernia and varicose vein repair. Patients who had undergone this type of surgery were chosen for inclusion in the study, as traditionally these patients would have been hospitalized for a number of days. This type of surgery took place in the chosen research setting on 3 days each week, usually with one patient on each general operation list of the day. For this reason, a convenience sample was used. The first 16 patients who agreed to be interviewed were 5 women and 11 men. Three of the men had had varicose vein repair and the other eight men had had a hernia repair. The five women had had their varicose veins repaired. In total, the sample consisted of eight patients who had had a hernia repair and eight patients who had had varicose vein repair. The ages of the patients in the sample ranged from 30 
to 71 years. The mean age of the sample was 50 years.

\section{Ethical approval}

Ethical approval was sought and obtained from the Local Research Ethics Committee. All the patients signed a consent form in which they agreed to be interviewed.

\section{Data collection}

Case studies have been described as a research approach that involves the in-depth examination of either an individual or a group of people (Nieswiadomy, 1993). This approach appeared to be the appropriate choice, as qualitative data were needed in order to find out what patients thought and felt about day case surgery. A semi-structured interview schedule was used to collect the data. G.P.M. interviewed two patients for a pilot study, and as this proved satisfactory she decided to proceed with the schedule.

The researcher, who was a nurse working in another part of the hospital, solicited the help of the discharge nurse to recruit the sample. This nurse explained the nature and purpose of the study to the patients and gave each of them an explanatory letter and a consent form to sign. Each patient who agreed to take part in the study returned the form to the researcher together with details of how they could be contacted. The researcher then contacted the respondent and asked where and when they would like to be interviewed. All the patients requested to be interviewed in their own homes. Each interview lasted between 30 and 40 minutes and was tape-recorded. These interviews took place between 1 and 4 weeks after surgery.

\section{Data analysis}

The data from this study have been interpreted using a descriptive theory in the most basic form, namely the factor-isolating form (Diers, 1979). This provided labels for the phenomena identified, with descriptive definitions of the terms used, thus enabling the researcher to examine "what was there' and 'how it exactly was'. The method used to analyse the data had the advantage of allowing trends of concepts and theories to be easily identified and to flow throughout the findings of the study. The data were transcribed and coded. A procedure known as data reduction then took place. This consists of 'selecting, focusing, simplifying, abstracting and transforming' the data (Miles and Humberman, 1994). To try to overcome the problem of bias in interpreting the data collected for this study, the transcriptions were given to an experienced colleague for them to perform the same procedure as the researcher for analysis of the data. The results of this consultative process demonstrated agreement about the major themes and important issues raised by the respondents.

\section{Results}

A number of categories were identified in this study, but for the purposes of this paper only the following areas will be highlighted.

\section{Choice of day case surgery}

It is evident that the majority of patients did not have a choice as to whether they had their surgery performed as a day case or as an in-patient. Indeed, some patients were 'told' that the operation would be carried out as a day case: 'They just said that, come in for a day, there was no choice'. Another respondent stated that 'day care was the only thing mentioned, I went down to see the consultant and he said we will have you in and do it in the day'.

However, it was also apparent from the patients' retrospective replies that they would have chosen day case surgery if they had been presented with a choice. 'Well he [the GP] brought the subject up .... I heard about this day surgery and I asked my GP about that, so I made a point then of asking the consultant ... because I do not think anybody likes going into hospital, I know I don't!'

One respondent first knew that she was having day case surgery when her appointment letter arrived. She commented that 'varicose veins isn't a huge operation". Another patient said 'I didn't consider that surgery as absolute major. I suppose in a way, considering after this type of operation they expect you to walk, I didn't mind at all coming out'. In some ways it could be argued that patients were relieved to have the operation scheduled as day surgery, as this reinforced their perception of it as being of minor significance.

Others had viewed day surgery as a way to ensure quick turn-around of beds: 'I thought it was a good idea, you know just go in and come out', or had relied on the expertise of the doctors to guide them: 'People in that position, in medical 
terms then, if they think it is adequate, efficient, I take their word for it'.

\section{Assessment for day case surgery}

Assessment of patients determines whether they are fit enough for day surgery and whether they will be able to cope in their surroundings on returning home. The following replies were recorded when patients were asked about assessments:

\section{Interviewer:}

Did they ask about your arrangements at home?

Mr A: No, no, only if I had someone there.

$\mathrm{Mr} \mathrm{C}$ had not been assessed before the operation. When he was asked if he had been consulted about whether his wife would be at home with him, he replied 'That is the only thing they did ask after I came out from surgery ... not before, after the operation, would there be anybody in the house'. His wife (who had just entered the room), added 'We didn't know what he would be like, if we had I would have obviously taken the following day off or arranged something in advance'.

Mrs L was asked about her home circumstances, but this was when she was in hospital: 'Yes, they asked that when I went in and I had a form to fill in'.

$\mathrm{Mr} \mathrm{N}$, when asked if he was assessed before going into hospital, replied, 'Yes, he asked me a few questions, if there was any sort of personal problems or difficulties'. However, he was not asked about his circumstances.

Mr J replied 'No, nothing, but they did ask if I had a responsible adult at home'.

Thus from the interviews with the patients it is clear that they had not been adequately assessed prior to going into hospital.

All the patients who were interviewed had been asked if they had a responsible adult who would be with them overnight after the operation. However, the nature of the social support had not been investigated, nor had the availability of their carers been questioned. In addition, there was insufficient opportunity for the patient to discuss their concerns with the professionals. If patients are to be cared for in the community, then it is important that professionals should enquire in more detail about patients' home and social circumstances.

\section{Information received before day case surgery}

When patients were asked what information they had received before going into hospital, and whether they had a clear idea of what to expect, their replies varied. One respondent who did not receive any information said 'Perhaps I should have asked, but he [the surgeon] was in a hurry'.

Another patient, on the day of the operation, was given the opportunity to ask the doctor questions. 'He said, have you got any questions? So I asked him, what was the procedure, what they would do, and he explained about the operation'.

One male respondent stated that he knew what was going to happen because he had seen a documentary about it on television: '[On] Horizon, there was a programme on about hernias and "net" repairs ... I told them [the doctors] I knew what they were going to do'. Other patients who had received little or no information did not appear to be concerned: 'I thought, well, they know what they are doing, the surgeons, it's your life in their hands if you like, you don't ask questions, do you?, 'No, I don't think so, not knowing what happens after!', 'Quite briefly, they asked me if there is anything I wanted to know, did I want to ask questions. I said no, not really, I know what it is all about, and I'm not interested, to be honest'.

Another patient had found the information himself: 'Because I knew somebody that had happened to have had the operation, he said "shave yourself before going in"'. One woman had asked the doctor in hospital what would happen, but felt, 'Perhaps I was just being too nosy, but I wanted somebody to turn around and tell me what they were going to do in layman's language, and I found that out by asking my practice sister and GP when I went back to surgery afterwards'. She went on to say 'I still do not know why they did the puncturing. I am assuming because if the vein is redundant they have to drain it of blood so that is still a bit of a mystery'.

These findings demonstrated that the patients received little or no information before going into hospital, and that most of the information that they were given appeared to be provided on the day of surgery. This information was insufficient to give them adequate psychological or social preparation to meet their personal requirements. More importantly, information about after-care and selfmanagement appeared to be lacking.

Primary Health Care Research and Development 2001; 2: 55-61 


\section{Back home}

The real test of whether the information that patients were given was sufficient was when they returned home. One of the women stated 'If they put it down [written instructions] when they tell you not to drive and things like that, or how long to keep your stocking on for and when you can take it off, and when you can have a bath. They did tell me when to have a bath, but I'm not sure about the others'.

Mr B stated that 'the district nurse wasn't sure of the instructions [she had been given], her instructions did not say whether she should change the bandages or leave them, so she rang the hospital'.

Mr O felt that 'in my case I needed more information .... I don't remember anyone asking me about the stairs, ... a little more guidance'.

There was also confusion about the visit of the district nurse. Mr G stated that 'you were given information about the community nurse, I thought the nurse was coming that night, then I thought be on the safe side, it must be tomorrow . . . there was a bit of a mix-up, because the next day nobody arrived'. After a number of telephone calls the nurse eventually arrived that evening.

Mrs L also experienced some confusion: 'They told me in the hospital that she [the district nurse] would be there that night, but she didn't come, next morning she came, but they phoned to apologize for the misunderstanding'.

The district nurse arrived at the house of one of the respondents after 10 p.m. The patient stated 'I had been waiting and waiting. We thought she'll never come now at this time of night. So I took the dogs out any way. I struggled down, I didn't get far, admittedly, and I got as far as the back gate', at which time the district nurse arrived.

It became apparent from analysis of this factor that when patients were at home, they were very much left to their own devices. Little information was given to them, and there was no continuity in the care provided by professionals. Patients needed more information/permission with regard to how to behave (e.g., when to return to work, what everyday activities to undertake). Without this information, patients were lacking in confidence.

The data obtained from the interviews demonstrated that there appears to be a lack of communication between the professionals in the hospital and those in the community. As described above, there appears to be confusion with regard to the timing of district nurses' visits. This could be due to poor communication, but it might also reflect a lack of district nurse resources.

\section{Overall view of day case surgery}

Patients' overall views of their experience of day case surgery varied. Mr A stated 'I think it is a good system ... if you can come out the same day it is marvellous, as long as people are fit enough'.

Mr F and Mr D also had the same view: "the thought of staying in hospital every night, not that it is not convenient for me, perish the thought I like to be on the go and be active in my own environment' and 'I would always go for home on the day'.

Mr C, who had his hernia repaired, felt that the operation was too major for day surgery: 'I should have never come out, I mean I like getting out of hospital as quick as I can and be home, but I think I should have stayed in for at least 2 days'.

Most patients liked the idea of not having to stay in hospital overnight and of being able to return to their own home. It therefore did not seem to matter to them too much if they had had a bad experience. In fact they did not even notice that some areas of their care could be improved in order to make their recovery a little smoother and more comfortable.

\section{Discussion}

\section{Limitations of the study}

The number of patients who were having day surgery performed limited the selection of the sample in that the only variables determined were the type of operation. Therefore the sample was one of convenience. It may be asked why other operations performed as day case surgery were not included. The reason was that such operations are not as invasive as the operations that had been performed on the patients who were included in this study.

\section{Conclusions}

Some of the findings of this study replicated the results reported in previous studies. The Audit Commission (1990) also found that only a small percentage of patients had received any information prior to going into hospital for day case surgery. Firth (1991) found that only half of the 
patients who fulfilled the research criteria had been told to expect pain after their operation.

It is evident from the findings that health care is not always as seamless and consistent as one is led to believe. The Community Care Act (Department of Health, 1990), aimed at encouraging patients to be cared for at home, can only be successful if provisions for health care are of a consistently high standard.

The patients in this study were given conflicting information and in some cases no information at all about the nature of the treatment to be received or the necessary components of after-care. If the patients had been included in the planning of their care then this situation might not have arisen.

The data obtained showed that a small minority of the patients who were interviewed felt that the operation they had undergone (hernia repair) was not suitable for day case surgery. The remaining patients, despite problems with lack of privacy and inadequate information, were satisfied that their operations had been performed according to the principles of day case surgery. They were also satisfied with the system and the care that they had received.

However, we would argue that it is unethical to overlook the perceived defects in the current system of day care and to accept the nature of patients' satisfaction at face value. The overall outcome of this study is positive, in so far as it has identified that patients prefer to be in hospital for the minimum possible time and to return to their home as soon as they can safely do so. These findings are obviously very encouraging, as the NHS moves towards a philosophy of caring for patients in the community. The less positive findings concern specific issues relating to operational factors which impinge on care and patient behaviour. Particular problems identified were that patients were confused about roles and behaviour, and about professional inputs to care (e.g., when the district nurse would be visiting).

It appeared from the study that the hospital day care unit and the patient's home were two separate units providing completely different types of care. It could be argued that they are indeed separate, but surely the care that patients receive should flow in a constant manner between the two locations. Although patients are individuals and their needs differ, the principles of seamless care should not differ, but rather they should provide a bridge across the 'gap' between institutional and community care.

\section{Recommendations}

Because of the relatively small size of the sample and the use of qualitative methodology, any recommendations that are made must, of course, be tentative. A study such as this provides a 'snapshot' of an event and how it was perceived by a specific group of individuals in one place at one time. However, it is likely that many people with experience of day case surgery either as professionals or as patients will recognize many of the issues raised and the feelings expressed.

Several recommendations can therefore be made on the basis of this study. The policy and procedures for day case surgery should be revisited at a strategic level, to ensure that policies, procedures and protocols address the issues of increasing patient choice, improving the provision of information, reviewing assessment procedures, improving staff education and training, researching the concepts of patient empowerment, the effects of care commodification, improved care management and concepts of 'seamless' care.

The following issues should be addressed at an operational level, by setting up an assessment clinic where patients can be fully screened for their health and social needs prior to day surgery. The assessment should include details of their home environment, and social support systems, and patients could be given written information about their operation, and the requirements for cooperation in care and after-care. The possibility should be considered that the district nurse might visit the patient at home and conduct the assessment, as after all the patient will be returning home under their care. At this point the patient could be given verbal and written information about their condition and its management, and they could also be 'put in the picture' about the procedure of day case surgery and their recuperative role (obviously this information would only be given if the patient is willing to accept this).

Other questions need to be asked, such as whether there are sufficient district nurse resources, and whether day case surgery is overloading the caseload of the district nurse.

The study also needs to be replicated with a larger sample. 


\section{Acknowledgements}

The authors would like to thank Mrs A. Kelly of the School of Health Sciences at University of Wales, Swansea, and the staff and patients of the hospital in which the study was undertaken.

\section{References}

Audit Commission 1990: A short cut to better services. Day Surgery in England and Wales. London: Audit Commission.

Avis, M. 1992: Do patients want a choice in surgery? Nursing Times 88, 61.

Bates, T. and Hamm, J. 1989: Data unto the day patient. Health Service Journal 99, 266.

Bysshe, J.E. 1988: The effects of giving information to patients before surgery. Nursing 30, 36-39.

Carrington, S. 1993: Quality assurance in day surgery. Journal of One Day Surgery 2, 15-19.

Copp, G. 1988: Intra-operative information and pre-operative visiting. Surgical Nurse December, 27-29.

Department of Health 1989: A study of the management and utilisation of the operating department (Bevan Report). London: HMSO
Department of Health 1990: Community Care Act. London: HMSO.

Diers, D. 1979: Research in nursing practice. Philadelphia, PA: J.B. Lippincott.

Fawcett-Henessy A. 1995: All right on the day? Nursing Standard $9,52$.

Firth, F. 1991: Pain after day surgery. Nursing Times 87, 72-76.

James, R. 1993: Night and day. Health Service Journal 103, 22-24.

Miles, A.B. and Huberman, A.M. 1994: An expanded source book. Qualitative data analysis. Cited in Appleton, J. 1995: Journal of Advanced Nursing 22, 993-997.

NHS 1995: NHS Magazine. Quarterly Review Issue 3, 5-8.

NHS Management Executive 1993: Day surgery - report by the Day Surgery Task Force. London: Department of Health.

Nieswiadomy, R.M. 1993: Foundation of nursing research, 2nd edn. New York: Appleton \& Lange.

Ong, L.M.L., Haes De, J.C.J.M., Hoos, A.M. and Llammes, F.B. 1995: Doctor-patient communication: a review of the literature. Social Science and Medicine 40, 903-18.

Royal College of Surgeons of England 1985: Guidelines for day care surgery. London: Royal College of Surgeons of England.

Webb, R. 1995: Pre-operative visiting from the perspective of the theatre nurse. British Journal of Nursing 4, 919-25.

Welsh Office 1999: Patient episode database for Wales. Cardiff: Welsh Office. 